\title{
Insufficient Humidification of Respiratory Gases in Patients Who Are Undergoing Therapeutic Hypothermia at a Paediatric and Adult Intensive Care Unit
}

\author{
Yukari Tanaka, ${ }^{1}$ Sachiko Iwata, ${ }^{1}$ Masahiro Kinoshita, ${ }^{1}$ Kennosuke Tsuda, ${ }^{1}$ \\ Shoichiro Tanaka, ${ }^{1}$ Naoko Hara, ${ }^{1}$ Ryota Shindou, ${ }^{1}$ Eimei Harada, ${ }^{1}$ Ryouji Kijima, ${ }^{2}$ \\ Osamu Yamaga, ${ }^{2}$ Hitoe Ohkuma, ${ }^{2}$ Kazuo Ushijima, ${ }^{3}$ Teruo Sakamoto, ${ }^{4}$ \\ Yushiro Yamashita, ${ }^{1}$ and Osuke Iwata ${ }^{1}$ \\ ${ }^{1}$ Department of Paediatrics and Child Health, Kurume University School of Medicine, Fukuoka, Japan \\ ${ }^{2}$ Department of Clinical Engineering, Kurume University Hospital, Fukuoka, Japan \\ ${ }^{3}$ Department of Anaesthesiology, Kurume University School of Medicine, Fukuoka, Japan \\ ${ }^{4}$ Advanced Emergency Medical Service Centre, Kurume University Hospital, Fukuoka, Japan
}

Correspondence should be addressed to Osuke Iwata; o.iwata@ucl.ac.uk

Received 10 December 2016; Revised 2 March 2017; Accepted 28 March 2017; Published 20 April 2017

Academic Editor: Emmanuel Charbonney

Copyright (c) 2017 Yukari Tanaka et al. This is an open access article distributed under the Creative Commons Attribution License, which permits unrestricted use, distribution, and reproduction in any medium, provided the original work is properly cited.

\begin{abstract}
For cooled newborn infants, humidifier settings for normothermic condition provide excessive gas humidity because absolute humidity at saturation is temperature-dependent. To assess humidification of respiratory gases in patients who underwent moderate therapeutic hypothermia at a paediatric/adult intensive care unit, 6 patients were studied over 9 times. Three humidifier settings, 37-default (chamber-outlet, $37^{\circ} \mathrm{C}$; Y-piece, $40^{\circ} \mathrm{C}$ ), 33.5-theoretical (chamber-outlet, $33.5^{\circ} \mathrm{C}$; Y-piece, $36.5^{\circ} \mathrm{C}$ ), and 33.5 -adjusted (optimised setting to achieve saturated vapour at $33.5^{\circ} \mathrm{C}$ using feedback from a thermohygrometer), were tested. Y-piece gas temperature/humidity and the incidence of high $(>40.6 \mathrm{mg} / \mathrm{L})$ and low $(<32.9 \mathrm{mg} / \mathrm{L})$ humidity relative to the target level $(36.6 \mathrm{mg} / \mathrm{L})$ were assessed. Y-piece gas humidity was 32.0 (26.8-37.3), 22.7 (16.9-28.6), and 36.9 (35.5-38.3) mg/L \{mean (95\% confidence interval)\} for 37-default setting, 33.5-theoretical setting, and 33.5-adjusted setting, respectively. High humidity was observed in 1 patient with 37-default setting, whereas low humidity was seen in 5 patients with 37-default setting and 8 patients with 33.5-theoretical setting. With 33.5-adjusted setting, inadequate Y-piece humidity was not observed. Potential risks of the default humidifier setting for insufficient respiratory gas humidification were highlighted in patients cooled at a paediatric/adult intensive care unit. Y-piece gas conditions can be controlled to the theoretically optimal level by adjusting the setting guided by Y-piece gas temperature/humidity.
\end{abstract}

\section{Introduction}

A number of clinical studies investigated the neuroprotective effect of therapeutic hypothermia to $32-34^{\circ} \mathrm{C}$ in a range of pathological conditions [1-5]. However, in part because of serious adverse events occurring during cooling, such as septicaemia and ventilator-associated pneumonia, it has been difficult to demonstrate the superiority of moderate hypothermia to conventional normothermic care or targeted temperature management to $36^{\circ} \mathrm{C}$ except for hypoxic-ischaemic encephalopathy in newborn infants [3-6]. An improved regimen, which reduces systemic adverse events during cooling, may significantly improve the outcome of patients who undergo therapeutic hypothermia at paediatric and adult intensive care units (ICUs).

For mechanically ventilated patients, respiratory gases are humidified close to saturated vapour at $37^{\circ} \mathrm{C}(43.9 \mathrm{mg} / \mathrm{L})$, regardless of body temperature [7]. However, under hypothermic conditions, absolute humidity at saturation decreases in a temperature-dependent manner. Therefore, the default humidifier setting for normothermic condition may provide excessive humidity and condensation in hypothermic 


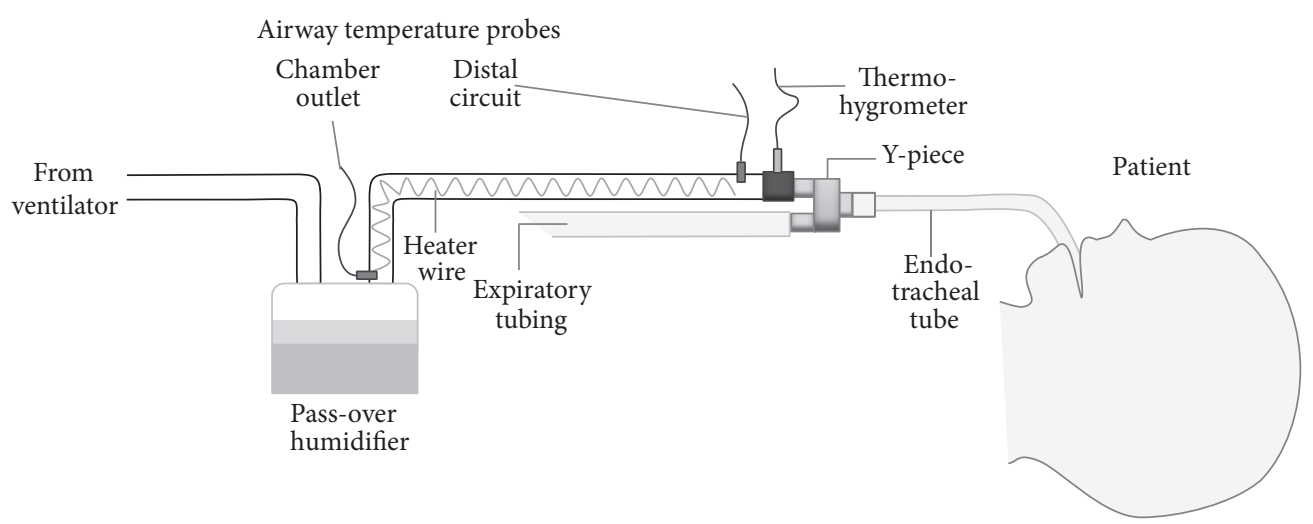

FIGURE 1: Configuration of the ventilator circuit. A thermohygrometer was inserted between the distal end of the heated inspiratory circuit and the Y-piece. Nonheated extension tubes were not used in the current study cohort.

patients [8-10]. Indeed, our previous study demonstrated that the default humidifier setting provided excessive gas heating and humidity in patients cooled at neonatal intensive care units [11]. Excessive humidification might be associated with the incidence of adverse events during cooling. However, the incidence of inadequate humidification of respiratory gases and its association with clinical adverse events remains unknown in patients cooled at paediatric and adult ICUs.

The aim of this study was to examine humidification of respiratory gases at the Y-piece of the ventilator circuit in patients of paediatric and adult ICUs who are undergoing therapeutic hypothermia.

\section{Materials and Methods}

This study was conducted under the approval of the Ethics Committee of Kurume University School of Medicine with informed consent from substitute decision-makers of the patients.

2.1. Study Population. Between July 2011 and November 2014, 3 children and 3 adults who underwent moderate hypothermia to $33-34^{\circ} \mathrm{C}$ at a tertiary emergency medical centre in Kurume, Fukuoka, Japan, were studied 9 times over different days (Table 1 ).

2.2. Ventilators, Circuits, and Humidifiers. Three ventilators (the 840, Covidien, Minneapolis, MN; VELA, CareFusion, San Diego, CA; and Evita XL, Dräger, Lübeck, Germany) were used according to the availability and the patient's condition. For these ventilators, oxygen and synthetic air are delivered from the central gas supply except for VELA, which compresses ambient air using its turbine. We used a pass-over humidifier (MR 730; Fisher and Paykel, Auckland, New Zealand) with ventilator-fixed circuits of either Dar (Covidien, for 840) or Evaqua 2 (Covidien, for VELA or Evita XL) (Figure 1).

2.3. Data Collection. Data collection from the same patient was repeated up to twice in total with an interval of at least $24 \mathrm{~h}$ only after major changes in ventilator settings. Gases
TABLE 1: Clinical background variables of the study population.

\begin{tabular}{lc}
\hline Variables & $\begin{array}{c}\text { Number }(\%), \text { median (range) } \\
\text { or median }\{95 \% \mathrm{CI}\}\end{array}$ \\
\hline Sex (female) & $3(50.0 \%)$ \\
Age (year) & $28.0(0.5-61.8)$ \\
Body weight $(\mathrm{kg})$ & $30.4(5.0-66.0)$ \\
Cooling indication & \\
Cardiopulmonary arrest & $3(50.0 \%)$ \\
Acute encephalopathy & $2(33.3 \%)$ \\
Subarachnoid haemorrhage & $1(16.7 \%)$ \\
Ventilator & \\
840 & $4(66.7 \%)$ \\
VELA & $1(16.7 \%)$ \\
Evita XL & $1(16.7 \%)$ \\
Ventilator mode & \\
Patient-triggered & $5(83.3 \%)$ \\
Bilevel & $1(16.7 \%)$ \\
Constant flow of ventilator $(\mathrm{L} / \mathrm{min})$ & $4.9\{3.3-6.6\}$ \\
Mean airway pressure $\left(\mathrm{cm} \mathrm{H}_{2} \mathrm{O}\right)$ & $10.7\{7.9-13.5\}$ \\
Fraction of inspired oxygen $(\%)$ & $0.5\{0.3-0.6\}$ \\
Ambient temperature $\left({ }^{\circ} \mathrm{C}\right)$ & $25.8\{24.9-26.8\}$ \\
Ambient humidity $(\%)$ & $37.6\{34.8-40.3\}$ \\
Core body temperature $\left({ }^{\circ} \mathrm{C}\right)$ & $34.2\{33.6-34.9\}$ \\
Size of endotracheal tube $(\mathrm{mm})$ & $5.5(4.0-9.0)$ \\
Adverse events & $1(16.7 \%)$ \\
Tube obstruction & $1(16.7 \%)$ \\
Pneumothorax & $2(33.3 \%)$ \\
Ventilator-associated pneumonia & 0 \\
Septicaemia & 0 \\
Death & \\
Days on ventilator & $(1-88)$ \\
\hline
\end{tabular}

were assessed using a clinical, main-stream capacitive thermohygrometer (Moiscope, Siynet, Tokyo, Japan; response time, $3 \mathrm{~s}$ within the range of 40 to $100 \%$; accuracy, $\pm 0.5^{\circ} \mathrm{C}$ 
for temperature and $\pm 3.5 \%$ for relative humidity) $[12,13]$ placed between the distal end of the inspiratory circuit and the Y-piece ("Y-piece" temperature and humidity) (Figure 1). Although this thermohygrometer is designed for continuous monitoring, we took out the sensor from the circuit to dry it up when studies persisted longer than 10 minutes, or when condensation was observed within the circuit adjacent to the sensor. Temperature values at the humidifying chamber outlet ("chamber outlet" temperature) were provided by the humidifier. Three humidifier settings were serially assessed as follows: (i) 37-default setting (default humidifier setting for normothermic patients; chamber outlet, $37^{\circ} \mathrm{C}$; Y-piece, $40^{\circ} \mathrm{C}$ ), (ii) 33.5-theoretical setting (theoretically adjusted humidifier setting for moderate hypothermia by lowering the target chamber outlet temperature and Y-piece temperature to $33.5^{\circ} \mathrm{C}$ and $36.5^{\circ} \mathrm{C}$, resp.), and (iii) 33.5 -adjusted setting (humidifier settings adjusted to deliver absolute humidity at saturation at $33.5^{\circ} \mathrm{C}$, i.e., a temperature of $36.5^{\circ} \mathrm{C} \pm 0.5^{\circ} \mathrm{C}$ and humidity of $36.6 \pm 0.5 \mathrm{mg} / \mathrm{L}$ or the closest values at the Ypiece). After the temperature (chamber outlet and Y-piece) and humidity (Y-piece) had become stable with each humidifier setting over 10 minutes, these variables were recorded three times every 3 minutes. The ambient temperature and humidity were also recorded at the beginning of data collection using a capacitive thermohygrometer $(605-\mathrm{Hl}$, Testo, Lenzkirch, Germany; accuracy, $\pm 0.5^{\circ} \mathrm{C}$ for temperature and $\pm 3 \%$ for relative humidity). Patients' clinical backgrounds, treatments, adverse events, and short-term outcomes were obtained from the clinical records.

2.4. Data Analysis. Values are presented as mean (95\% confidence interval) unless otherwise stated. Because of the lack of established thresholds for inappropriate Y-piece gas temperature and humidity during cooling, high and low temperature/humidity was defined based on clinical recommendations for normothermic patients that Y-piece temperature should be controlled to $\pm 2^{\circ} \mathrm{C}$ from the target level, with its humidity $>75 \%$ of saturated vapour at body temperature [7]. Thus, the incidence of high temperature $>38.5^{\circ} \mathrm{C}$ (i.e., 36.5 plus 2.0 ), low temperature $<34.5^{\circ} \mathrm{C}$ (i.e., 36.5 minus 2.0 ), high humidity $>40.6 \mathrm{mg} / \mathrm{L}$ \{saturated vapour at $35.5^{\circ} \mathrm{C}$ (i.e., 33.5 plus 2.0$)\}$, low humidity $<32.9 \mathrm{mg} / \mathrm{L}$ \{saturated vapour at $31.5^{\circ} \mathrm{C}$ (i.e., 33.5 minus 2.0 ) $\}$, and extremely low humidity $<27.4 \mathrm{mg} / \mathrm{L}\left\{75 \%\right.$ of saturated vapour at $\left.33.5^{\circ} \mathrm{C}\right\}$ was assessed for each humidifier setting.

\section{Results}

Six patients of 28.0 (0.5 to 61.8) \{median (range)\} years old were studied nine times over different days. Three children used tracheal tubes of $4-5.5 \mathrm{~mm}$ in diameter, whereas three adults were intubated with tracheal tubes of $7-9 \mathrm{~mm}$ in diameter. Seven and two studies were performed in patients who were ventilated using patient-triggered ventilation and bilevel ventilation, respectively. A turbine air ventilator was used in an adult patient with the fraction of inspiratory oxygen of 0.21 when ambient temperature and humidity were $25.9^{\circ} \mathrm{C}$ and $36.6 \%$, respectively (patient 6).
3.1. Adverse Events. A 5-month-old infant (patient 1) developed pneumothorax and ventilator-associated pneumonia during cooling and required chest drainage and intravenous antibiotics (Tables 1 and 2). Another 61-year-old patient (patient 6) developed ventilator-associated pneumonia during cooling, which was successfully treated using intravenous antibiotics. Tracheal tube obstruction was observed in a 5year-old child (patient 3) $16 \mathrm{~h}$ after the commencement of cooling. Y-piece gas temperature and humidity were assessed shortly after reintubation and were $39.9^{\circ} \mathrm{C}$ and $28.9 \mathrm{mg} / \mathrm{L}$, respectively, with the 37 -default setting.

3.2. Setting of 37-Default. Y-piece gas temperature and humidity were $39.1(38.5-39.8)^{\circ} \mathrm{C}$ and $32.0(26.8-37.3) \mathrm{mg} / \mathrm{L}$, respectively. High temperature, high humidity, low humidity, and extremely low humidity were observed in 7, 1, 5, and 2 studies, respectively (Table 2).

3.3. Setting of 33.5-Theoretical. Y-piece gas temperature and humidity were $36.4(36.1-36.7)^{\circ} \mathrm{C}$ and $22.7(16.9-28.6) \mathrm{mg} / \mathrm{L}$, respectively. Low humidity and extremely low humidity were observed in 8 and 6 studies, respectively.

3.4. Setting of 33.5-Adjusted. Humidifier settings for the chamber outlet and the Y-piece temperature were 37.9 $(36.6-39.2)^{\circ} \mathrm{C}$ and $37.5(37.0-38.0)^{\circ} \mathrm{C}$, respectively (Table 2). Y-piece gas temperature and humidity were $37.5(37.1-38.0)^{\circ} \mathrm{C}$ and 36.9 (35.5-38.3) mg/L, respectively. High/low Y-piece temperature and humidity were not observed except for an infant (patient 1) whose Y-piece temperature was $38.6^{\circ} \mathrm{C}$.

\section{Discussion}

Use of the default humidifier setting for normothermic conditions in patients cooled at paediatric and adult ICU resulted in higher temperature and lower humidity of Y-piece gases compared with the target levels for hypothermic conditions. This finding contrasted with our previous study in newborn infants, where both Y-piece temperature and humidity were higher than the target levels. Such a trend towards insufficient humidification in the current ICU cohort was exacerbated when the theoretically adjusted humidifier setting for body temperature was used and not observed when the humidifier setting was adjusted according to the Y-piece gas monitoring.

4.1. Temperature and Humidity of Respiratory Gases during Therapeutic Hypothermia. For mechanically ventilated patients, respiratory gases are prepared till they become saturated vapour at $37^{\circ} \mathrm{C}(43.9 \mathrm{mg} / \mathrm{L})$ or at least $75 \%$ of this level $(32.9 \mathrm{mg} / \mathrm{L})$ [7]. Even during therapeutic hypothermia, where absolute humidity at saturation considerably reduces, the default humidifier setting for the normothermic condition is currently recommended. We initially anticipated that the use of this setting in cooled patients may lead to excessive humidification and condensation, which may theoretically cause adverse events, such as mucosal dysfunction and airway oedema $[9,14]$. Indeed, our previous study in cooled newborn infants highlighted the common incidence of excessive Ypiece gas humidification when this default humidifier setting 


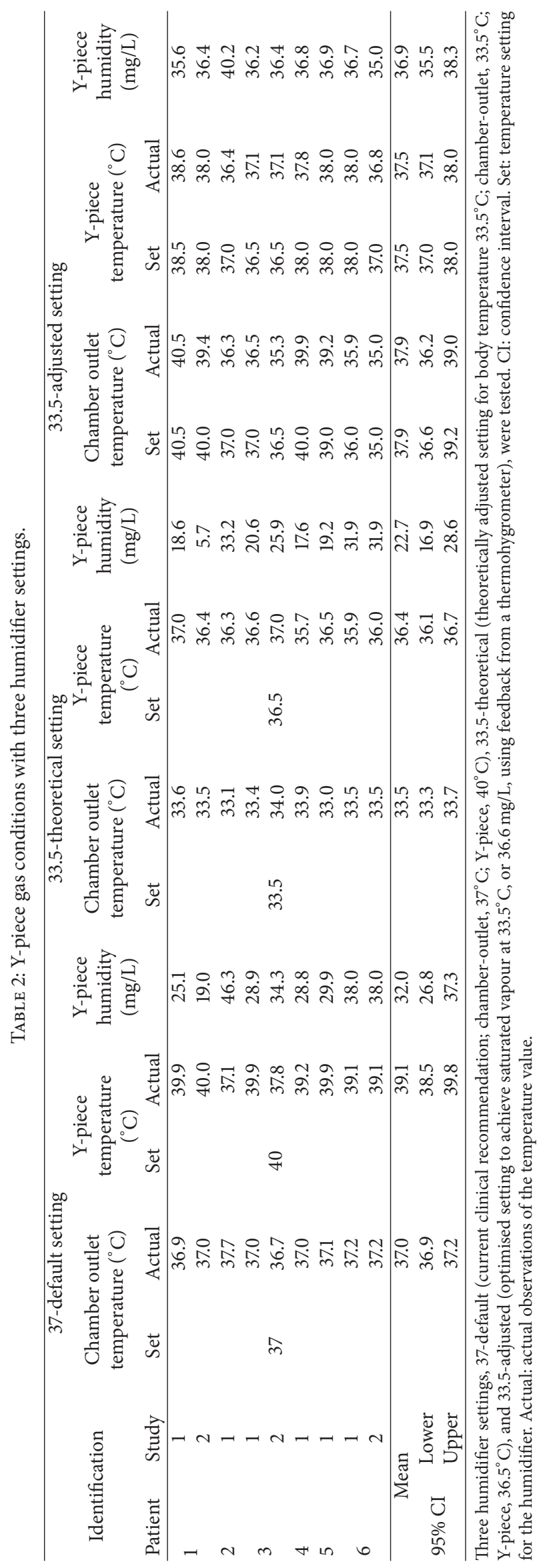


for normothermia was used [11]. In the current study, with the 37-default setting, Y-piece gas temperature was higher and humidity was lower than the target levels. Subsequently, with the 37-default setting, Y-piece gas humidity was lower for the current ICU cohort by $7.2 \mathrm{mg} / \mathrm{L}$ in average than the level observed in newborn infants despite similar temperature levels [11]. Incidence of systemic complications, such as endotracheal tube obstruction, atelectasis, and ventilatorassociated pneumonia, might be investigated in patients, who undergo therapeutic hypothermia at paediatric and adult ICUs, in relation to insufficient humidification of respiratory gases.

\subsection{Mechanism of Insufficient Humidification in Cooled} Children and Adults. The exact mechanism of insufficient humidification in the current ICU cohort remains unknown. Current humidifying systems generally provide only modest humidity as opposed to the ideal level $[15,16]$. In addition, insufficient humidity of gases has been reported in association with elevated gas temperature at the inlet of the humidifying chamber $[17,18]$. When respiratory gases are already heated by the ventilator and are close to $37^{\circ} \mathrm{C}$, the heating power of the humidifier is turned off, where only the temperature, but not the humidity, is prepared for the target level. This is consistent to the current observation of insufficient Y-piece gas humidity despite its paradoxically higher temperature, as well as the exacerbation of insufficient humidification with the 33.5-theoretical setting. However, considering that these mechanisms are likely to affect newborn, child, and adult patients equally, contrasting observations of the Y-piece gas humidity between these cohorts would be attributed to the difference in the detailed ventilator settings, environmental factors, and the patient's clinical variables. For example, compared to newborn infants, respiratory care for child and adult patients would be characterised by the use of balloon-cuffed tracheal tubes of relatively larger diameters, and relatively greater constant flow of the ventilator. Turbine air ventilators, such as VELA in our study, are known to provide relatively warmer respiratory gases, and subsequent humidity of respiratory gases may further be affected by the fraction of inspiratory oxygen and ambient temperature/humidity $[19,20]$. The impact of these variables on the Y-piece gas humidity needs to be assessed in future studies by incorporating temperature and humidity monitoring at the chamber inlet and outlet in a sufficient number of child and adult patients.

\subsection{Potential Solutions for Insufficient Humidification of} Respiratory Gases. Our previous and current study suggested that the Y-piece gas condition can be significantly altered by ventilator settings and patients' clinical conditions, suggesting the difficulty in predicting and preventing the incidence of inappropriate humidification of respiratory gases, especially in cooled patients. Nevertheless, when the humidifier setting was adjusted by obtaining feedback from Y-piece gas monitoring, high and low humidification was not observed. The humidifier used in the current study (MR 730) has been replaced by its successor model (MR 850), which provides fully automated adjustment of respiratory gases using feedback from energy consumption and gas flow, resulting in relatively more efficient humidification of gases $[15,18]$. The use of MR 850 may reduce the incidence of insufficient gas humidification during cooling, however, with simultaneously increased risks for excessive humidification. Given that the temperature setting of MR 850 cannot be manually adjusted, solution for problems might be difficult. Our previous study suggested that a counter-flow humidifier, which passes gases to a water shower [21], provided perfect temperature/humidity control for cooled newborn infants. The benefit of using the counter-flow humidifier needs to be assessed in further large-scale studies.

\section{Limitations}

There currently is a lack of evidence to support the safety and benefit of a particular strategy in preparing respiratory gases during therapeutic hypothermia. Similarly, there is no established threshold to define inappropriate gas temperature and humidity for cooled patients. In our current study, thresholds for inappropriate Y-piece gas temperature and humidity were defined based on the recommendations released by the International Organization for Standardization for normothermic patients [7], which might be different from the ones associated with clinical adverse events. Our study cohort is too small to confirm their risks and problems. For the same reason, we were unable to highlight the risk factors for inappropriate humidification during cooling. These limitations need to be considered when interpreting our findings to clinical practice.

\section{Conclusions}

Humidification of respiratory gases was different from the level, which are estimated from the humidifier setting in patients who were undergoing therapeutic hypothermia at paediatric and adult ICUs. With the default humidifier setting for normothermic patients, insufficient gas humidification was observed, the trend of which was exacerbated with the humidifier setting adjusted for the hypothermic body temperature. This is the first report to warn of the potential risk of inappropriate humidification in child and adult patients who are undergoing therapeutic hypothermia under mechanical ventilation. When the humidifier setting was adjusted with the feedback from the Y-piece gas temperature and humidity, respiratory gases were successfully humidified to the theoretical target level for hypothermic patients. Future studies are needed to confirm the incidence of inappropriate humidification during cooling in ICU patients and to test whether a new regimen with optimal gas preparation reduces ventilator-associated pneumonia and other serious adverse events encountered during therapeutic hypothermia.

\section{Conflicts of Interest}

The authors have indicated that they have no financial relationships relevant to this article to disclose. 


\section{Authors' Contributions}

Yukari Tanaka, Masahiro Kinoshita, Kennosuke Tsuda, Sachiko Iwata, and Osuke Iwata conceptualised the study. Shoichiro Tanaka, Naoko Hara, Ryota Shindou, Eimei Harada, Ryouji Kijima, Osamu Yamaga, Hitoe Ohkuma, Masahiro Kinoshita, Kennosuke Tsuda, Kazuo Ushijima, Teruo Sakamoto, Yushiro Yamashita, and Osuke Iwata contributed to patient recruitment and data collection. Sachiko Iwata, Masahiro Kinoshita, and Osuke Iwata performed statistical analysis. Yukari Tanaka, Sachiko Iwata, and Osuke Iwata drafted the manuscript. Masahiro Kinoshita, Kennosuke Tsuda, Kazuo Ushijima, Teruo Sakamoto, and Yushiro Yamashita critically reviewed and revised the manuscript. All authors have seen and approved the final version of the manuscript.

\section{Acknowledgments}

The authors thank Dr. Hiroki Saitsu and Ms. Chiho Yoshii for their assistance and encouragement. Osuke Iwata was funded by the Japan Science and Technology Agency and the Ministry of Education, Culture, Sports, Science and Technology (Grant-in-Aid for Scientific Research C16K09005). Sachiko Iwata was funded by the Japan Science and Technology Agency and the Ministry of Education, Culture, Sports, Science and Technology (Grant-in-Aid for Scientific Research C15K09733).

\section{References}

[1] The Hypothermia after Cardiac Arrest Study Group, "Mild therapeutic hypothermia to improve the neurologic outcome after cardiac arrest," New England Journal of Medicine, vol. 346, no. 8, pp. 549-556, 2002.

[2] D. V. Azzopardi, B. Strohm, A. D. Edwards et al., "Moderate hypothermia to treat perinatal asphyxial encephalopathy," New England Journal of Medicine, vol. 361, no. 14, pp. 1349-1358, 2009.

[3] G. L. Clifton, A. Valadka, D. Zygun et al., "Very early hypothermia induction in patients with severe brain injury (the National Acute Brain Injury Study: Hypothermia II): a randomised trial," The Lancet Neurology, vol. 10, no. 2, pp. 131-139, 2011.

[4] J. S. Hutchison, R. E. Ward, J. Lacroix et al., "Hypothermia therapy after traumatic brain injury in children," New England Journal of Medicine, vol. 358, no. 23, pp. 2447-2456, 2008.

[5] M. M. Todd, B. J. Hindman, W. R. Clarke, and J. C. Torner, "Mild intraoperative hypothermia during surgery for intracranial aneurysm," New England Journal of Medicine, vol. 352, no. 2, pp. 135-145, 2005.

[6] N. Nielsen, J. Wetterslev, T. Cronberg et al., "Targeted temperature management at $33^{\circ} \mathrm{C}$ versus $36^{\circ} \mathrm{C}$ after cardiac arrest," New England Journal of Medicine, vol. 369, no. 23, pp. 2197-2206, 2013.

[7] ISO, "Respiratory tract humidifiers for medical use. Particular requirements for respiratory humidification systems," Tech. Rep. 8185, ISO, 2007, http://www.iso.org/iso/catalogue_detail .htm?csnumber $=38527$ (Last accessed in 21 Nov 2016).

[8] J. J. Pillow, N. H. Hillman, G. R. Polglase et al., "Oxygen, temperature and humidity of inspired gases and their influences on airway and lung tissue in near-term lambs," Intensive Care Medicine, vol. 35, no. 12, pp. 2157-2163, 2009.
[9] T. M. Sottiaux, "Consequences of under- and over-humidification," Respiratory Care Clinics of North America, vol. 12, pp. 23352, 2006.

[10] R. Williams, N. Rankin, T. Smith, D. Galler, and P. Seakins, "Relationship between the humidity and temperature of inspired gas and the function of the airway mucosa," Critical Care Medicine, vol. 24, no. 11, pp. 1920-1929, 1996.

[11] S. Tanaka, S. Iwata, M. Kinoshita et al., "Use of normothermic default humidifier settings causes excessive humidification of respiratory gases during therapeutic hypothermia," Therapeutic Hypothermia and Temperature Management, vol. 6, no. 4, pp. 180-188, 2016.

[12] D. Inui, J. Oto, and M. Nishimura, "Effect of heat and moisture exchanger (HME) positioning on inspiratory gas humidification," BMC Pulmonary Medicine, vol. 6, article 19, 2006.

[13] H. Wada, H. Higuchi, and S. Arimura, "Temperature and humidity of the Dräger Cato anesthetic machine circuit," Journal of Anesthesia, vol. 17, no. 3, pp. 166-170, 2003.

[14] F. Lellouche, S. Qader, S. Taille, A. Lyazidi, and L. Brochard, "Under-humidification and over-humidification during moderate induced hypothermia with usual devices," Intensive Care Medicine, vol. 32, no. 7, pp. 1014-1021, 2006.

[15] P. Pelosi, D. Chiumello, P. Severgnini et al., "Performance of heated wire humidifiers: An In Vitro study," Journal of Critical Care, vol. 22, no. 3, pp. 258-264, 2007.

[16] H. S. Al Ashry and A. M. Modrykamien, "Humidification during mechanical ventilation in the adult patient," BioMed Research International, vol. 2014, Article ID 715434, 12 pages, 2014.

[17] B. G. Carter, N. Whittington, M. Hochmann, and A. Osborne, "The effect of inlet gas temperatures on heated humidifier performance," Journal of Aerosol Medicine: Deposition, Clearance, and Effects in the Lung, vol. 15, no. 1, pp. 7-13, 2002.

[18] F. Lellouche, S. Taillé, S. M. Maggiore et al., "Influence of ambient and ventilator output temperatures on performance of heated-wire humidifiers," American Journal of Respiratory and Critical Care Medicine, vol. 170, no. 10, pp. 1073-1079, 2004.

[19] Y. Chikata, M. Onodera, H. Imanaka, and M. Nishimura, "Temperature of gas delivered from ventilators," Journal of Intensive Care, vol. 1, no. 1, p. 6, 2013.

[20] F. Lellouche, S. M. Maggiore, A. Lyazidi, N. Deye, S. Taillé, and L. Brochard, "Water content of delivered gases during non-invasive ventilation in healthy subjects," Intensive Care Medicine, vol. 35, no. 6, pp. 987-995, 2009.

[21] S. Schumann, C. A. Stahl, K. Möller, H.-J. Priebe, and J. Guttmann, "Moisturizing and mechanical characteristics of a new counter-flow type heated humidifier," British Journal of Anaesthesia, vol. 98, no. 4, pp. 531-538, 2007. 


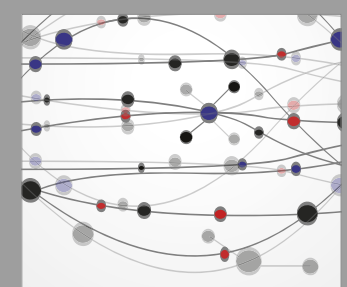

The Scientific World Journal
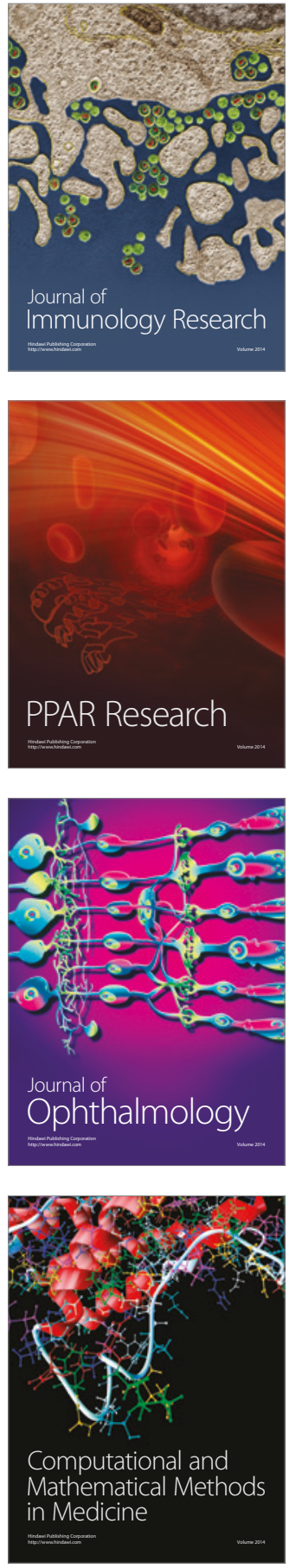

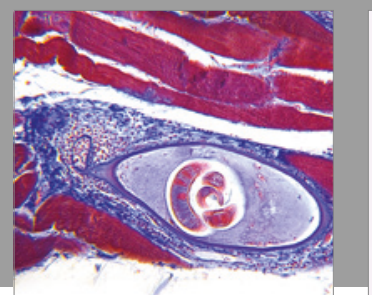

Gastroenterology Research and Practice
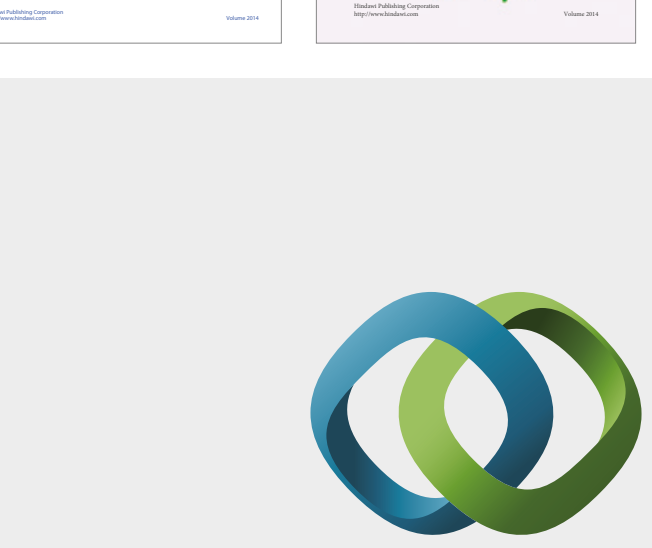

\section{Hindawi}

Submit your manuscripts at

https://www.hindawi.com
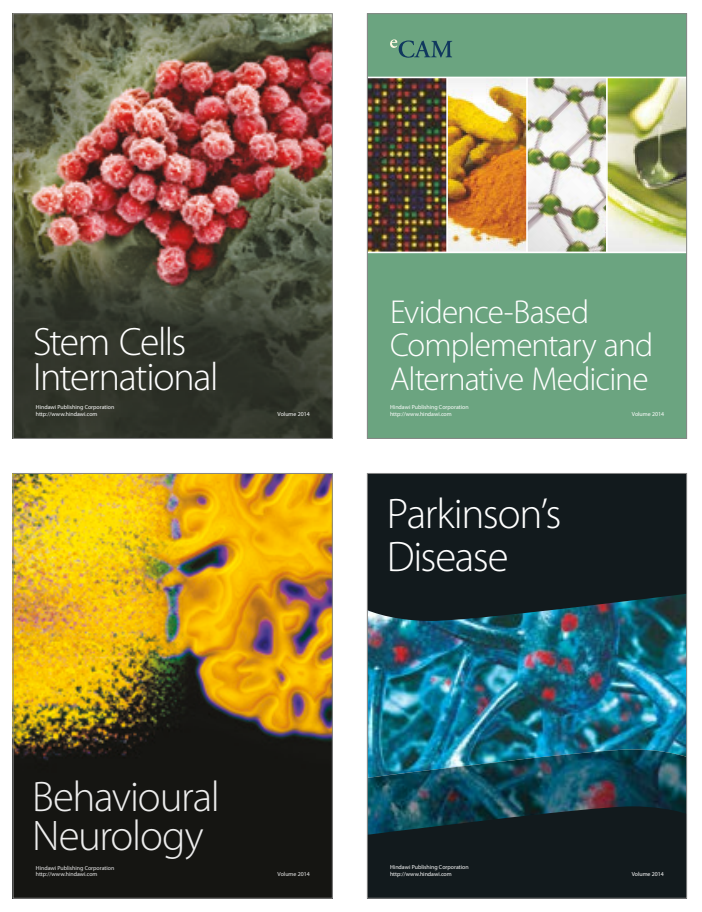
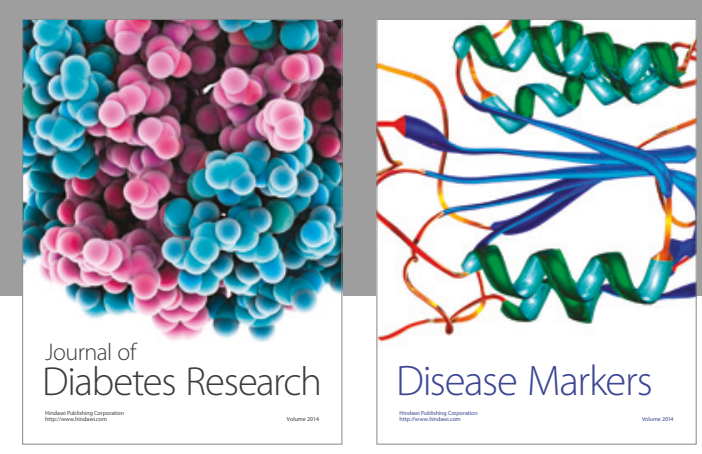

Disease Markers
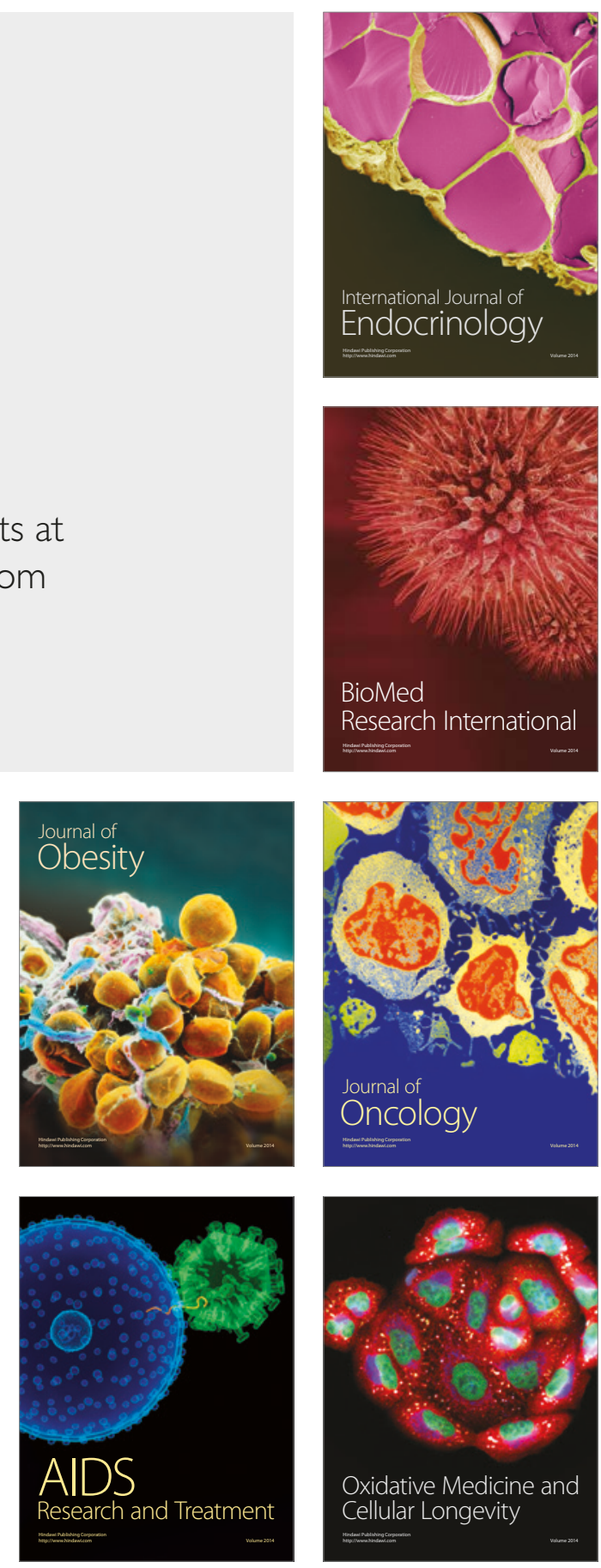